\title{
0 Leito Placentário no Descolamento Prematuro da Placenta
}

\author{
The Placental Bed in Abruptio Placentae
}

Maria Rita de Souza Mesquita ${ }^{1}$, Nelson Sass ${ }^{1}$, João Norberto Stavalle ${ }^{2}$, Luiz Camano ${ }^{1}$

\section{RESUMO}

Objetivo: análise histopatológica das artérias espiraladas do leito placentário em gestações complicadas pelo descolamento prematuro da placenta (DPP) associado à hipertensão, comparando-as com a estrutura vascular dos leitos placentários normais.

Método: a biópsia do leito placentário foi realizada em 23 gestantes com diagnóstico de descolamento prematuro de placenta associado à hipertensão (G/HA) e idade gestacional maior ou igual a 28 semanas, submetidas ao parto cesáreo. O grupo controle (GC) foi constituido por 30 pacientes, sem doenças, submetidas a parto cesáreo por indicação obstétrica. As variáveis histológicas selecionadas para estudo foram: padrão inalterado, modificações fisiológicas, desorganização da camada média, alterações hiperplásicas, necrose e aterose aguda.

Resultados: nas pacientes com DPP associado à hipertensão ocorreu uma predominância significativa de desorganização da camada média, detectada em 50\% das pacientes, e de alterações hiperplásicas, em comparação ao GC, ao passo que a presença de modificações fisiológicas foi estatisticamente mais significante no GC. Achados como necrose e aterose aguda foram observados em menores proporções no G/HA, mas sem diferenças significantes entre os dois grupos.

Conclusões: os achados histológicos vasculares predominantes em grávidas com diagnóstico de DPP associado à hipertensão foram desorganização da camada média e alterações hiperplásicas. A presença do padrão patológico foi significativamente maior no G/HA, sendo o mais prevalente a desorganização da camada média. Houve predomínio do padrão normal, isto é, modificações fisiológicas no GC.

PALAVRAS-CHAVE: Descolamento prematuro da placenta. Circulação placentária. Gravidez normal. Pré-eclâmpsia. Hipertensão.

\section{Introdução}

Definido pela Organização Mundial da Saúde ${ }^{1}$ como a separação súbita da placenta normalmente inserida no corpo uterino, após a vigésima semana de gestação, o descolamento prematuro da placenta (DPP), também denominado abruptio placentae, representa causa importante entre as sindromes hemorrágicas da segunda metade da gestação.

${ }^{1}$ Departamento de Obstetrícia, ${ }^{2}$ Departamento de Patologia, Universidade Federal de São Paulo/Escola Paulista de Medicina, São Paulo, SP

Correspondência:

Maria Rita de Souza Mesquita

R. Duarte de Carvalho, 221, apto 42

03084-030 - São Paulo - SP

Fone/Fax: (11) 3663-4286

e-mail: mrsmesquita@hotmail.com
O DPP é dramático evento que acomete 0,5 a $3,0 \%$ das gestações, elevando a morbidade materna, além de ser responsável por mais de um quarto de todos os óbitos perinatais ${ }^{2}$. Para Cunningham et $a .^{3}$, sua incidência e severidade têm diminuído progressivamente nos últimos anos.

Há evidências consideráveis sugerindo que os estados hipertensivos da gestação constituem o principal fator etiopatogênico envolvido no $\mathrm{DPP}^{3,4}$. Pritchard et al. ${ }^{5}$ relataram que a hipertensão arterial materna estaria relacionada a $50 \%$ dos quadros graves de DPP. Segundo Camano ${ }^{6}$, a hipertensão arterial crônica (HAC) com vasculopatia seria a grande causa de DPP, enquanto o aparecimento deste quadro com a doença hipertensiva específica da gravidez ocorreria excepcionalmente.

Até 1958, os estudos morfológicos sobre placentação humana normal e patológica eram 
baseados na análise histológica da placenta pósparto, na avaliação do útero pós-histerectomia ou por meio de necrópsia e traziam limitações, tanto metodológicas quanto clínicas, dificultando a compreensão da circulação uteroplacentária ${ }^{7}$. Foi importante a introdução de nova técnica para a obtenção de fragmentos do útero gravídico durante o parto cesáreo, denominada biópsia do leito placentário, descrita por Dixon e Robertson ${ }^{7}$ em 1958, enfatizando a presença obrigatória de decídua basal, assim como de miométrio subjacente contendo a origem das artérias uteroplacentárias.

Por meio da biópsia do leito placentário, Brosens et al. ${ }^{8}$ descreveram a ocorrência de duas ondas de invasão trofoblástica retrógrada dos vasos uterinos. A primeira, que ocorreria até a $12^{a}$ semana, é caracterizada pela substituição do tecido muscular e elástico das artérias espiraladas por material fibrinóide, somente em sua porção decidual. A segunda onda de invasão trofoblástica ocorreria entre a $14^{\mathrm{a}}$ e a $21^{\mathrm{a}}$ semana, envolvendo agora os segmentos intramiometriais, tornandoos condutos fetalizados, bastante dilatados, denominando-as artérias uteroplacentárias, com melhores condições de manter os niveis de oxigenação fetal. Relataram, ainda, que estas modificações fisiológicas seriam detectadas logo após a nidação, em máximas proporções no leito placentário, área representativa do sítio de implantação, e, em minimas proporções, nas áreas periféricas da placenta madura.

Segundo Robertson et al. ${ }^{9}$, a presença de distúrbios hipertensivos manteria as artérias espiraladas com parede muscular integra e sem vasodilatação, por não terem desenvolvido a segunda onda de invasão trofoblástica e conseqüente redução do débito sangüíneo uteroplacentário. Os autores concluiram, a partir deste estudo, que, embora a hipertensão arterial essencial ocasione lesões morfológicas sistêmicas tardiamente, também induz à proliferação do tecido conectivo nas paredes das artérias basais, além de determinar proliferação excessiva do tecido fibroso e muscular liso, tanto na intima como na média, promovendo estenose ou até oclusão das mesmas. Em pacientes com préeclâmpsia ( $\mathrm{PE})$ e eclâmpsia $(\mathrm{E})$, estes autores observaram necrose fibrinóide aguda e uma segunda lesão, constituída por dano na parede do vaso associado à presença de células lipídicas, chamadas de ateromatose aguda. Verificaram, ainda, que em face de HAC com PE sobreposta, as artérias do leito placentário apresentavam uma combinação de lesões proliferativas e necróticas ${ }^{9}$.

Apesar de o DPP estar associado, em grande parte das vezes, aos estados hipertensivos, muitos aspectos sobre sua real etiologia ainda estão obscuros ${ }^{3}$. A probabilidade de existir lesão primária nos vasos placentários não pode ser descartada $^{10}$.

Assim, nasceu a nossa motivação de estudar os aspectos histopatológicos dos vasos uteroplacentários em gestações complicadas pelo descolamento placentário, com o desiderato de contribuir para o melhor entendimento da fisiopatologia desta grave enfermidade obstétrica.

\section{Pacientes e Métodos}

Este estudo descritivo teve como finalidade a análise dos padrões histopatológicos das artérias do leito placentário em gestações complicadas pelo DPP associado à hipertensão, comparando-os com a estrutura vascular dos leitos placentários normais. Os critérios de inclusão foram gestações com diagnóstico de DPP, com indicação de parto cesáreo e idade gestacional maior ou igual a 28 semanas. Foram excluidas pacientes com condições clínicas como diabete melito, infecções agudas e colagenoses; doenças obstétricas como placenta prévia e gemelaridade; antecedentes de doenças tromboembólicas e os casos com DPP que tiveram resolução por via vaginal. O grupo controle (GC) foi constituído por 30 pacientes com evolução gestacional normal, de termo e submetidas a parto cesáreo por indicação obstétrica.

O DPP foi definido pela separação inopinada, intempestiva e prematura da placenta normalmente inserida, após a $20^{a}$ semana de gestação ${ }^{6}$. O diagnóstico de DPP baseou-se em evidências clínicas de dor abdominal súbita de intensidade variável, geralmente associado à hipertonia uterina e a sangramento vaginal ou a hemorragia oculta, com ou sem sinais de estado hipovolêmico. Quando possível, a visualização ultra-sonográfica de áreas anecóicas retroplacentárias sugestivas de hematoma ou ainda a detecção durante o ato cirúrgico de coágulo retroplacentário e/ou presença de cratera na face materna da placenta foram considerados para a caracterização diagnóstica.

O quadro hipertensivo foi caracterizado pela constatação de pressão arterial sistólica igual ou maior a $140 \mathrm{mmHg}$ e/ou pressão arterial diastólica igual ou maior a $90 \mathrm{mmHg}$, ao longo da internação. Levando-se em conta as características clinicas do DPP, todos os quadros hipertensivos foram compactados na mesma categoria e denominados estados hipertensivos.

A biópsia do leito placentário foi realizada 
segundo técnica descrita por Robertson et al. ${ }^{11}$. O local de inserção placentária foi identificado pelo cirurgião logo após o nascimento do concepto. Após a extração da placenta, a inspeção minuciosa do sítio de implantação permitiu identificar o leito placentário propriamente dito, localizando-se a zona mais próxima possivel ao centro da implantação. Um fragmento em forma de cunha de aproximadamente $1,0 \mathrm{~cm}$ de diâmetro em seu maior eixo foi obtido com bisturi lâmina 15. Quando necessário, realizou-se um ponto hemostático com fio absorvivel. O fragmento da biópsia foi submetido à fixação em formalina a $10 \%$ e encaminhado para um único patologista, que realizou a análise do material, sem o conhecimento do quadro clínico em questão.

O espécime foi inicialmente submetido a exame macroscópico sistemático, aferindo-se os diâmetros longitudinal, ântero-posterior e transverso. Posteriormente foi desidratado em concentrações crescentes de etanol, submetido a diafanização em xilol e incluído em parafina por período médio de 12 horas. Foram realizados cortes teciduais seriados com espessura aproximada de três micrômetros e as lâminas coradas pela técnica de hematoxilina e eosina. Para a melhor identificação das fibras elásticas e colágenas foram utilizados o método de tricrômio de Masson e o de Verhoeff ${ }^{12}$. Na presença de necrose utilizamos a coloração de hematoxilina fosfotúngstica ${ }^{12}$. Em seguida, os cortes histológicos foram examinados ao microscópio óptico.

As amostras foram consideradas representativas do leito placentário em sua porção central quando foram detectadas a presença de trofoblasto extraviloso na decídua e no miométrio, a predominância de células gigantes multinucleadas e a presença de no mínimo uma artéria espiralada em sua porção decidual e miometrial ${ }^{11}$.

Os padrões histológicos foram definidos, segundo a padronização de Meekins et al. ${ }^{13}$, em: padrão inalterado: quando as artérias permanecem morfologicamente normais ou semelhantes à estrutura não gravídica, ou seja, com as camadas intima, média e adventícia preservadas quanto à espessura e características histológicas; modificações fisiológicas: quando as artérias espiraladas apresentam invasão trofoblástica endovascular. O grau de invasão trofoblástica é determinado pela extensão de material fibrinóide detectado por técnicas de coloração padronizadas (Figura 1); desorganização da camada média: caracterizada por fibrose e hialinização na parede vascular (Figura 2); alterações hiperplásicas da camada íntima e média: definidas pelo espessamento da camada intima, formando camadas concêntricas de tecido fibroelástico e hialinização da camada média; necrose: caracterizada pela perda celular e eosinofilia intensa de todas as camadas; aterose aguda: determinada pela necrose fibrinóide, hialinização da parede vascular e infiltrado de histiócitos xantomizados na subíntima (Figura 3).

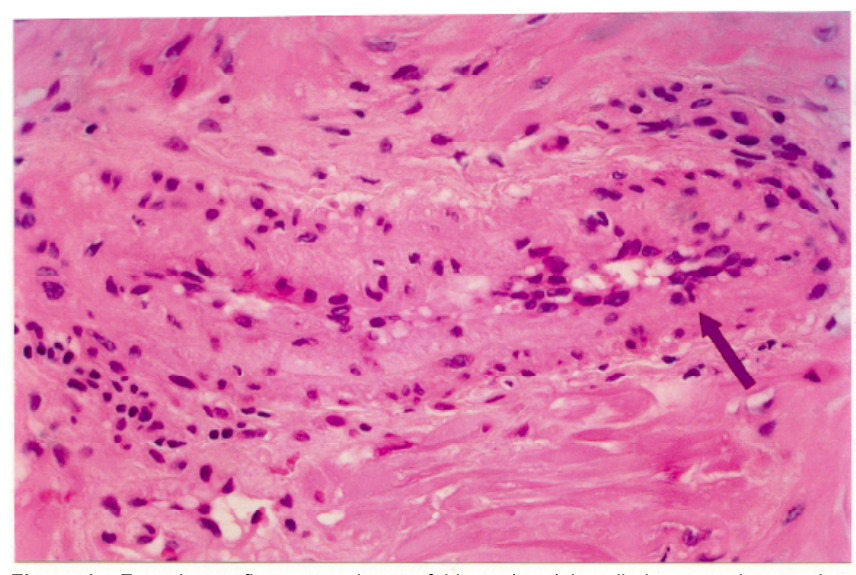

Figura 1 - Fotomicrografia mostrando o trofoblasto (seta) invadindo a parede vascular. (HE, 400X).

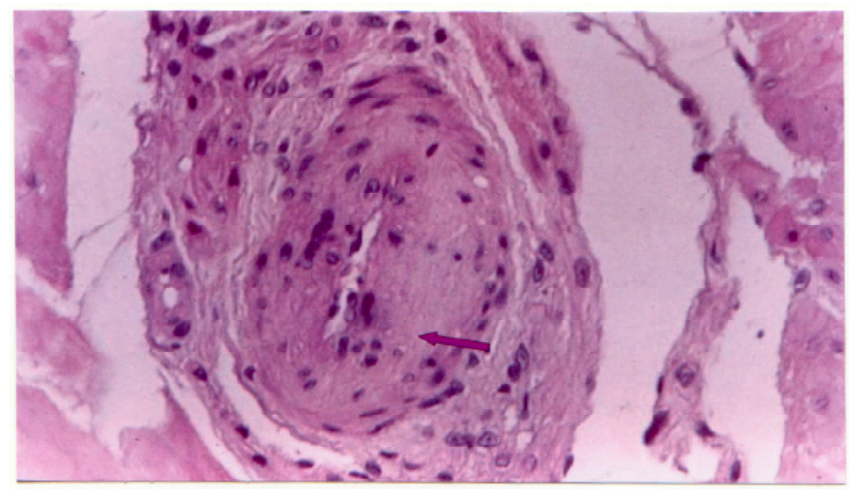

Figura 2 - Fotomicrografia mostrando espessamento fibroso focal (seta) da parede vascular (HE, 400X).

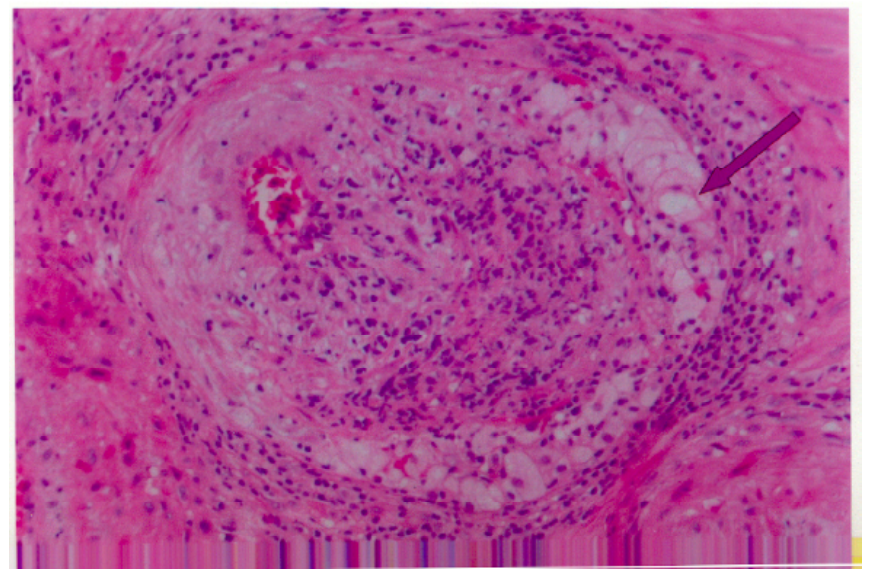

Figura 3 - Fotomicrografia mostrando grande quantidade de macrófagos xantomizados (seta) na subíntima, com oclusão quase total da luz vascular (HE, 400X). 
O padrão normal foi definido pela presença do padrão inalterado e de modificações fisiológicas, ao passo que desorganização da camada média, alterações hiperplásicas, necrose e aterose aguda foram consideradas como padrões patológicos.

Foi utilizado o teste da decomposição aditiva do $\chi^{2}{ }^{14}$ obedecendo-se às restrições de Cochran para a avaliação de possiveis contingências entre a presença ou ausência de modificações fisiológicas, desorganização da camada média, hiperplasia das camadas intima e média, necrose e aterose entre os grupos. Em todos os casos, o nivel de rejeição para a hipótese de nulidade foi fixado sempre em um valor menor ou igual a 0,05 $(5 \%)$. O presente estudo foi aprovado pelo Comitê de Ética em Pesquisa da Universidade Federal de São Paulo/Hospital São Paulo - CEP nº 0397/02.

\section{Resultados}

Para este estudo foram selecionadas 53 pacientes, sendo 23 acometidas pelo DPP associado a distúrbios hipertensivos (G/HA) e 30 pertencentes ao GC. Foram obtidos cinqüenta e três fragmentos a partir de biópsias do leito placentário. Todos eles tiveram os diâmetros longitudinal, ântero-posterior e transverso medidos após fixação no formol. O volume médio dos fragmentos foi de $1,03 \mathrm{~cm}^{3}$.

Na Tabela 1 podemos comparar os diferentes padrões histológicos segundo a classificação descrita por Meekins et al. ${ }^{13}$ para os grupos G/HA e GC. Nas pacientes com DPP associado à hipertensão ocorreu uma predominância de desorganização da camada média e de hiperplasia das camadas íntima e média em comparação ao GC. Em contrapartida, a presença de modificações fisiológicas nas artérias espiraladas do GC foi estatisticamente maior. Denotamos pequenas proporções de necrose e aterose no G/HA, sem significância estatística na comparação.

Tabela 1 - Padrões histológicos das artérias espiraladas no leito placentário nas pacientes com descolamento prematuro da placenta associado à hipertensão e no grupo controle.

\begin{tabular}{lrrr}
\hline Padrão histológico & $\begin{array}{c}\text { G/HA } \\
\mathbf{n}\end{array}$ & $\begin{array}{c}\text { GC } \\
\text { n }\end{array}$ & $\mathbf{p}$ \\
\hline Padrão inalterado & 2 & 9 & 0,057 \\
Modificação fisiológica & 2 & 20 & $<0,001^{*}$ \\
Desorganização da camada média & 15 & 2 & $<0,001^{*}$ \\
Hiperplasia das camadas íntima e média & 8 & 0 & $<0,001^{*}$ \\
Necrose & 2 & 0 & 0,184 \\
Aterose aguda & 2 & 0 & 0,184 \\
\hline Teste do $\chi^{2}$ ou teste exato de Fisher & \multicolumn{3}{c}{ G/HADPP - associado à hipertensão } \\
GC - grupo controle & ${ }^{*}$-significante & \\
& \multicolumn{4}{l}{}
\end{tabular}

A presença de padrões histológicos considerados patológicos, isto é, desorganização da camada média, hiperplasia das camadas íntima e média, necrose e aterose, foi significativamente maior no G/HA $(82,6 \%)$ em comparação a $6,7 \%$ no GC. Esta diferença é significante, com $\chi^{2}$ calculado de 28,2 (Tabela 2).

Tabela 2 - Comparação entre os achados histológicos das artérias espiraladas considerando o padrão normal ou patológico, nas pacientes com descolamento prematuro da placenta associado à hipertensão e no grupo controle.

\begin{tabular}{lrcrc}
\hline Padrão histológico & \multicolumn{2}{c}{ G/HA } & \multicolumn{2}{c}{ GC } \\
& $\mathbf{n}$ & $\%$ & $\mathbf{n}$ & $\%$ \\
\hline Normal & 4 & 17,4 & 28 & $93,3^{*}$ \\
Patológico & 19 & $82,6^{*}$ & 2 & 6,7 \\
Total & 23 & 100 & 30 & 100 \\
\hline $\begin{array}{l}\text { G/HA - DPP associado à hipertensão } \\
\text { GC - Grupo controle }\end{array}$ & $\begin{array}{l}p<0,001 \\
{ }^{*} \text {-significante }\end{array}$ & $\chi_{\text {calc. }}^{2}=28,29$ & \\
\end{tabular}

A lesão vascular predominante foi desorganização da camada média, detectada em $50 \%$ das pacientes. No que tange ao GC, a análise histopatológica das artérias espiraladas revelou predominância estatisticamente significativa de modificações fisiológicas $(93,3 \%)$ e do padrão inalterado.

\section{Discussão}

Há muito, em nossas reflexões, estamos motivados a compreender os aspectos envolvidos no determinismo do DPP, pois em nossa óptica, este tema clássico ainda apresenta aspectos etiológicos não devidamente esclarecidos. Esta preocupação é plenamente justificável, em face das múltiplas repercussões que ocasiona, tanto para o concepto quanto para a mãe.

Para a coleta de amostra tecidual satisfatória, é imprescindivel a identificação correta da área que corresponde ao sítio placentário propriamente dito. O leito placentário, ou seja, porção decidual subjacente à face materna da placenta, pode ser facilmente reconhecido como área com focos de depressão sangrante e friável. Assim como Robertson et al. ${ }^{11}$, observamos que a dificuldade técnica de biópsia somente ocorre quando a inserção placentária se localiza na parede uterina anterior, sendo necessário promover discreta eversão desta área para a extração do fragmento.

Outro fator relevante estaria relacionado aos critérios para caracterizar o fragmento tissular 
como próprio do leito placentário. A presença de trofoblasto é a única evidência que confirma a derivação do leito placentário. Além disso, a capacidade migratória do trofoblasto em converter gradualmente as artérias espiraladas em uteroplacentárias ocorre, em maiores proporções, nas áreas centrais do sítio placentário, não necessariamente em todas as artérias ou mesmo em todos os segmentos de sua extensão. Não denotamos complexidade em realizar o procedimento de biópsia na área mais central do sítio de implantação. Apenas um de todos os fragmentos obtidos foi excluído do estudo em decorrência de volume insatisfatório. Os demais apresentaram volume médio de $1,03 \mathrm{~cm}^{3}$ e englobaram os critérios mínimos de validação da amostra, isto é, presença de trofoblasto e artéria espiralada em sua porção decidual e miometrial.

Em contrapartida, Voigt e Becker ${ }^{10}$ e Dommisse e Tiltman ${ }^{15}$ relataram, respectivamente, a exclusão de 23,2 e 34,4\% das amostras colhidas, por não serem representativas do leito placentário. Acreditamos que biópsia realizada com técnicas inadequadas isto é, nas áreas periféricas do sítio de implantação e/ou em profundidade menor ou igual a $0,5 \mathrm{~cm}$, teriam maior probabilidade de serem insuficientes para representar todo o tecido placentário, além de apresentar distribuição irregular do trofoblasto neste nivel. Julgamos que a identificação minuciosa da área central do sítio de implantação, assim como a exérese de fragmento com $1,00 \mathrm{~cm}^{3}$ de diâmetro em média, são critérios considerados suficientes para a obtenção de uma amostra representativa.

Em nosso estudo, a análise dos padrões histológicos mostrou menor proporção de modificações fisiológicas nas artérias espiraladas nos G/HA quando comparados individualmente ao GC. Segundo Pijnenborg et al. ${ }^{16}$, a proporção de artérias espiraladas acometidas por modificações fisiológicas é progressivamente maior até o termo, sugerindo que a invasão trofoblástica destes vasos não está confinada aos primeiros dois trimestres da gestação. Estes autores ressaltaram que a biópsia do leito placentário somente pode ser obtida no final da gestação, dificultando esclarecimentos se a inibição trofoblástica precede, acompanha ou segue o desenvolvimento da hipertensão na gravidez.

Para Domisse e Tiltman ${ }^{15}$, das 12 pacientes que desenvolveram o descolamento placentário, 5 apresentaram modificações fisiológicas, ao passo que das 7 que não apresentaram tais modificações, quatro eram hipertensas. Assim como Robertson et al. ${ }^{9}$, acreditamos que a ocorrência das duas ondas de invasão trofoblástica esteja di- retamente relacionada à ausência de distúrbio hipertensivo. Assim, quando ocorre o DPP em pacientes com hipertensão associada, a possibilidade de não ter ocorrido a segunda onda de invasão trofoblástica na artéria espiralada é consideravelmente maior ${ }^{9}$.

Os mesmos autores relataram a presença de espessamento das camadas intima e subintima com morfologia similar às descritas por Brosens et al. ${ }^{8}$, em $41,7 \%$ da biópsias do leito placentário no DPP, provavelmente resultantes de trombose prévia ou, até mesmo, em resposta à turbulência normal do fluxo sangüíneo encontrada no segmento miometrial das artérias espiraladas livres da transformação fisiológica. Como estas lesões são habitualmente encontradas em gestações normais, não são consideradas específicas do DPP.

$\mathrm{Na}$ nossa casuística, os achados histológicos compativeis com desorganização da camada média e hiperplasia das camadas íntima e média foram evidenciados em proporções significativas nos descolamentos placentários com hipertensão associada, o mesmo não ocorrendo em relação ao GC. Pijnenborg et al. ${ }^{16}$ e Meekins et al. ${ }^{17}$ descreveram as características histológicas das artérias espiraladas no leito placentário em gestações normais e naquelas complicadas por hipertensão, concluindo que a ausência de modificações fisiológicas pode não ser peculiar da $\mathrm{PE}$, mas, possivelmente, está associada às várias formas de hipertensão na gravidez, mesmo que a paciente não desenvolva o descolamento placentário. Ressaltaram, ainda, que lesões histológicas como hiperplasia e desorganização da camada média ao nivel da porção miometrial da artéria espiralada, além da aterose aguda, também estão freqüentemente associadas aos distúrbios hipertensivos.

Meekins et al. ${ }^{13}$ demonstraram que o tratamento hipotensor em gestações complicadas por hipertensão crônica é mais benéfico para o organismo materno e não favorece melhor adaptação fisiológica das artérias espiraladas e nem diminui os riscos de complicações fetais relacionadas às alterações vasculares. Além disso, relataram que a terapêutica medicamentosa não reduz a ocorrência de desorganização, hiperplasia e aterose vascular no leito placentário.

Lesões histológicas compativeis com necrose e aterose foram detectadas, em nosso estudo, por duas vezes dentre as pacientes acometidas pelo DPP associado a síndromes hipertensivas, porém sem significância estatística. Para Hertig ${ }^{18}$, certas lesões patológicas são caracterizadas pelo acúmulo de gordura e leucócitos mononucleares abaixo da íntima das artérias espiraladas e degeneração fibrinóide da camada média seguida da 
substituição fibroblástica da íntima, levando à oclusão completa da luz vascular ${ }^{18}$. Na tentativa de explicar a gênese do DPP, o autor concluiu que a obstrução dos vasos que nutrem o endométrio levaria à necrose da decídua ao seu redor, induzindo a hemorragia que, em proporções crescentes, ocasionaria o descolamento placentário. No estudo realizado por Hertig ${ }^{18}$, estas lesões compatíveis com aterose não se mostraram patognomônicas do DPP associado às síndromes hipertensivas da gestação, estando, provavelmente, relacionadas mais aos distúrbios vasculares ou renais prévios que propriamente ao descolamento $^{18}$. É possivel que os espasmos vasculares das artérias espiraladas, sem lesões morfológicas demonstradas, tenham um papel ainda mais relevante na gênese do descolamento.

Dentre as pacientes hipertensas acometidas pelo DPP, Domisse e Tiltman ${ }^{15}$ descreveram a presença de um caso de aterose aguda na artéria uteroplacentária, em sua porção miometrial, com acúmulo de lipófagos e depósito de fibrina na camada média. Para Lewis e Benirschke ${ }^{19}$, a principal doença decidual, também chamada de aterose do leito vascular decidual, caracteriza-se pela necrose e hialinização da parede vascular acoplada ao depósito de macrófagos xantomizados, geralmente presentes em duas condições maternas: pressão sangüinea materna elevada e necrose vascular decidual ocasionada por vasculite ou infecção bacteriana decidual.

Em nossa casuística, os dois achados de aterose aguda estiveram relacionados às formas graves de hipertensão e a idades gestacionais mais precoces. Embora não seja a alteração mais freqüente, podemos aferir que se trata de lesão histológica diretamente interligada a danos vasculares graves e a desfechos materno-fetais ominosos. Ainda que outros estudos microscópicos analisando os vasos do leito placentário sejam necessários para definir a significância destas lesões no determinismo do DPP, estamos convencidos de que sua presença represente importante valor prognóstico em gestações futuras.

Quando agrupamos os achados histológicos em padrões normal ou patológico, denotamos que as alterações vasculares patológicas apresentaram proporções significativamente maiores no G/ HA que no GC (Tabela 2). Resultados semelhantes foram aferidos por Voigt e Becker ${ }^{10}$, demonstrando maior prevalência de aspectos histomorfológicos considerados patológicos em pacientes com niveis tensionais elevados. Estas afirmações reforçam a premissa de que no DPP associado a HA, as alterações vasculares podem ser histologicamente associadas à doença de base.
Estamos convencidos de que o determinismo do DPP esteja intimamente ligado às modificações estruturais da parede vascular diante de distúrbios hipertensivos. Assim, quando levamos em conta sua tendência em recidivar, isto pode sugerir que tais alterações histológicas vasculares possam ter valor prognóstico em futuras gestações. Em contrapartida, a ausência de alterações vasculares placentárias exalta a necessidade de novos estudos revendo outras causas possivelmente associadas à etiopatogenia do DPP.

No epílogo de nossas considerações cumpre ressaltar que a biópsia do leito placentário traz subsídios importantes até para avaliar o futuro obstétrico. É de se imaginar que as pacientes que apresentam alterações vasculares relevantes teriam evoluções gestacionais associadas a prognósticos reservados.

\section{ABSTRACT}

Purpose: to analyze the histopathological patterns of the placental bed arteries in pregnancies complicated by abruptio placentae (AP) and compare them with the normal vascular histology of the placental bed.

Methods: placental bed biopsy was performed in 23 pregnant women with a diagnosis of abruptio placentae associated with high blood pressure (G/HBP) disorders, with gestational age of 28 weeks or more, submitted to cesarean section. The control group (CG) consisted of 30 patients without disease, submitted to cesarean section for obstetric reasons. The selected histological variables were: unaltered pattern, physiological changes, medial layer disorganization, hyperplastic changes, acute necrosis and atherosis.

Results: in patients with AP associated with HBP there was a significant predominance of medial layer disorganization and hyperplastic changes, compared to $C G$, while physiological changes in spiral arteries were statistically more common in CG. Findings of acute necrosis and atherosis were observed in a low number of G/HBP, with no statistical significance.

Conclusions: in pregnant women with AP associated with $H B P$ the predominant vascular histological findings were medial layer disorganization and hyperplastic changes. The presence of histopathological features was significantly higher in $G / H B P$, with prevalence of medial layer disorganization. Normal histological pattern, i.e., physiological changes, were more prevalent in $C G$.

KEYWORDS: Abruptio placentae. Placental circulation. Normal pregnancy. Hypertension 


\section{Referências}

1. WHO: recommended definitions, terminology and format for statistical tables related to the perinatal period and use of a new certificate for cause of perinatal deaths. Modifications recommended by FIGO as amended October 14, 1976. Acta Obstet Gynecol Scand 1977; 56:247-53.

2. Witlin AG, Saade GR, Mattar F, Sibai BM. Risk factors for abruptio placentae and eclampsia: analysis of 445 consecutively managed women with severe preeclampsia and eclampsia. Am J Obstet Gynecol 1999; 180:1322-9.

3. Cunningham FG, Gant NF, Leveno KJ, Gilstrap LC, Hauth JC, Wenstrom KD. Obstetrical hemorrhage. In: Williams Obstetrics. $21^{\text {st }}$ ed. New York: McGrawHill; 2001. p.619-69.

4. Sibai BM. Chronic hypertension in pregnancy. Obstet Gynecol 2002; 100:369-77.

5. Pritchard JA, Cunningham FG, Pritchard SA, Mason RA. On reducing the frequency of severe abruptio placentae. Am J Obstet Gynecol 1991; 165:1345-51.

6. Camano L. Descolamento prematuro da placenta. In: Neme B, editor. Obstetrícia Básica. $2^{\mathrm{a}}$ ed. São Paulo: Sarvier; 2000. p.674-89.

7. Dixon HG, Robertson WB. A study of vessels of the placental bed in normotensive and hypertensive women. J Obstet Gynaecol Br Emp 1958; 65:803-9.

8. Brosens I, Robertson WB, Dixon HG. The physiological response of the vessels of the placental bed to normal pregnancy. J Pathol Bacteriol 1967; 93:569-79.

9. Robertson WB, Brosens I, Dixon HG. The pathological response of the vessels of the placental bed to hypertensive pregnancy. J Pathol Bacteriol 1967; 93:581-92.
10.Voigt HJ, Becker V. Doppler flow measurements and histomorphology of the placental bed in uteroplacental insufficiency. J Perinat Med 1992; 20:139-47.

11.Robertson WB, Khong TY, Brosens I, De Wolf F, Sheppard BL, Bonnar J. The placental bed biopsy: review from three European centers. Am J Obstet Gynecol 1986; 155:401-12.

12. Michalany J. Métodos selecionados de colorações e de impregnações argênticas. In: Michalany J, editor. Técnica Histológica em Anatomia Patológica: com instrução para cirurgião, enfermeira e citotécnica. $3^{a}$ ed. São Paulo: Michalany; 1998. p.125-81.

13.Meekins JW, Pijnenborg R, Hanssens M, McFadyen IR, van Assche A. Spiral artery morphology in pregnancies complicated by chronic hypertension: the relation to antihypertensive therapy and to superimposed preeclampsia. Hypertens Pregnancy 1995; 14:67-80.

14.Sidney S. Estatística não-paramétrica. $2^{a}$ ed. São Paulo: McGraw-Hill; 1975.

15.Dommisse J, Tiltman AJ. Placental bed biopsies in placental abruption. Br J Obstet Gynaecol 1992; 99:651-4.

16.Pijnenborg R, Anthony J, Davey DA, et al. Placental bed spiral arteries in the hypertensive disorders of pregnancy. Br J Obstet Gynaecol 1991; 98:648-55.

17.Meekins JW, Pijnenborg R, Hanssens M, McFadyen IR, van Assche A. Immunohistochemical identification of placental bed biopsies and the implications for the inclusion of specimens when studying the spiral artery response to pregnancy. Hypertens Pregnancy 1994; 13:61-9.

18. Hertig AT. Vascular pathology in hypertensive albuminuric toxemias of pregnancy. Clinics 1945; 4:602-14.

19.Lewis SH, Benirschke K. Placenta. In: Sternberg SS, editor. Histology for Pathologists. $2^{\text {nd }}$ ed. Philadelphia: Lippincott; 1997. p.961-94.

Recebido em: 21/8/2003 Aceito com modificações em: 16/9/2003

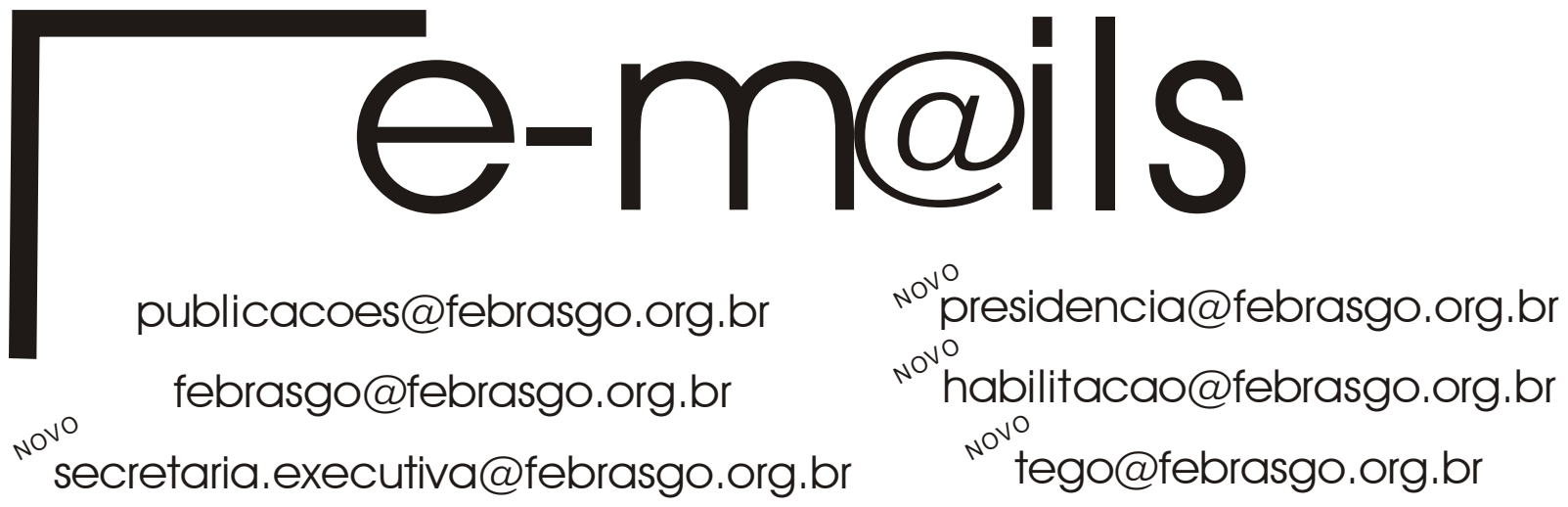

\section{DÚVIDAS - SUGESTÕES - ESCLARECIMENTOS MANDE SEU E-MAIL}

